\title{
LHCb silicon detectors: the Run 1 to Run 2 transition and first experience of Run 2
}

\author{
Kurt Rinnert* on behalf of the LHCb VELO \& ST Groups \\ University of Liverpool \\ E-mail: kurt.rinnertecern.ch
}

\begin{abstract}
$\mathrm{LHCb}$ is a dedicated experiment to study New Physics in the decays of heavy hadrons at the Large Hadron Collider (LHC) at CERN. The detector includes a high precision tracking system consisting of a silicon-strip vertex detector (VELO) surrounding the pp interaction region, a largearea silicon-strip detector located upstream of a dipole magnet (TT), and three stations of siliconstrip detectors (IT) and straw drift tubes placed downstream (OT). The operational transition of the silicon detectors VELO, TT and IT from LHC Run 1 to Run 2 and first Run 2 experiences will be presented. During the long shutdown of the LHC the silicon detectors have been maintained in a safe state and operated regularly to validate changes in the control infrastructure, new operational procedures, updates to the alarm systems and monitoring software. In addition, there have been some infrastructure related challenges due to maintenance performed in the vicinity of the silicon detectors that will be discussed. The LHCb silicon detectors are well prepared for LHC Run 2 and have already recorded tracks from injection line tests and low energy collisions. The results obtained from analyzing this data and the current status and plans for new operational procedures of the LHCb silicon detectors required in LHC Run 2 will be outlined.
\end{abstract}

24th International Workshop on Vertex Detector -VERTEX2015-

1-5 June 2015

Santa Fe, New Mexico, USA

\footnotetext{
* Speaker.
} 


\section{Introduction}

The LHCb detector [1][2] is a single-arm forward spectrometer covering the pseudorapidity range $2<\eta<5$, designed for the study of particles containing $b$ or $c$ quarks. The detector includes a high-precision tracking system consisting of a silicon-strip vertex detector surrounding the $p p$ interaction region [3], a large-area silicon-strip detector located upstream of a dipole magnet with a bending power of about $4 \mathrm{Tm}$, and three stations of silicon-strip detectors and straw drift tubes [7] placed downstream of the magnet. This paper focusses on the silicon detectors in the LHCb tracking system, namely the VELO (Vertex Locator), the TT (Tracker Turicensis) and the IT (Inner Tracker). The latter two are collectively designated the ST detectors due to their similar technology, layout and operation. The location of the silicon detectors inside the LHCb spectrometer is highlighted in Figure1.

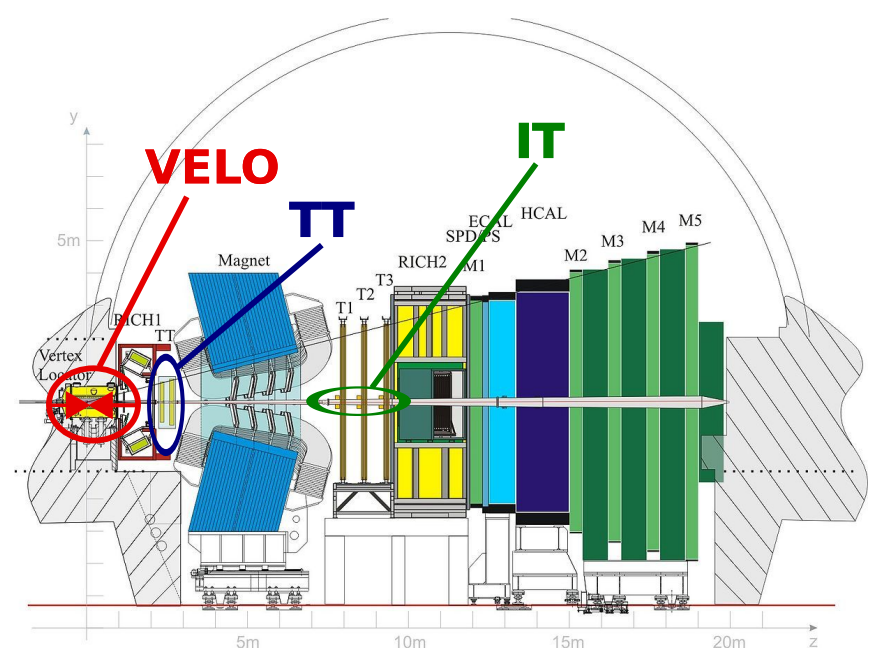

Figure 1: The LHCb spectrometer with the silicon detectors VELO, TT and IT highlighted.

The LHCb silicon detectors have been operated very successfully and efficiently during Run 1 of the LHC [3][5][6]. As is common, opportunities to further improve the robustness of detector operations and slow control configuration have been identified during the first phase of operations. In the interest of minimizing the risk of any impact on data taking efficiency, the implementation of these improvements had to wait until the LHC LS (Long Shutdown) 1. Improvements to the cooling and positioning systems of the silicon detectors, controls and configurations planned for and implemented during LS 1 will be described in the following sections. Furthermore, LHC Run 2 will impose new challenges in terms of the radiation damage accumulated by the silicon detectors; in particular by the VELO due to its extreme proximity to the interaction point. The new procedures to deal with these challenges will be outlined. A spare VELO has been built a few years ago in order to be ready for the unlikely event of a catastrophic accident or an unexpectedly long extension of LHC Run 2 [4].

\section{The Vertex Locator (VELO)}

The LHCb Vertex Locator (VELO) is unique in many respects among the silicon detectors 
deployed at LHC experiments. The detector operates extremely close to the LHC proton beams with the innermost strips being as close as $8.2 \mathrm{~mm}$ to the beam line. To protect the detector during the injection of LHC beams, it is comprised of two retractable detector halves that rest in their parking position of $\pm 3 \mathrm{~cm}$ from the beam line until stable beams are declared. Once the beams are stable the detector halves are moved in to center around the interaction region, using vertex measurements obtained by the detector itself, see Figure 3. The VELO operates in a secondary vacuum separated from the LHC vacuum by a $300 \mu \mathrm{m}$ thick aluminum foil, see Figure 2 . The VELO is cooled by a bi-phase $\mathrm{CO}_{2}$ cooling system. During normal operation, when the detector services are fully powered, the silicon sensors are typically at $-10^{\circ} \mathrm{C}$. The VELO is comprised of 21 modules per half, with one $R$-measuring and one $\phi$-measuring sensor each. In addition there are four pile-up (PU) veto $R$-type-only modules installed at the upstream end of the VELO. Each sensor has 2048 strips with strip pitch varying from $40 \mu \mathrm{m}$ to $100 \mu \mathrm{m}$. This results in an optimal single-hit resolution of $4 \mu \mathrm{m}$, depending on the incident angle of the charged particle.

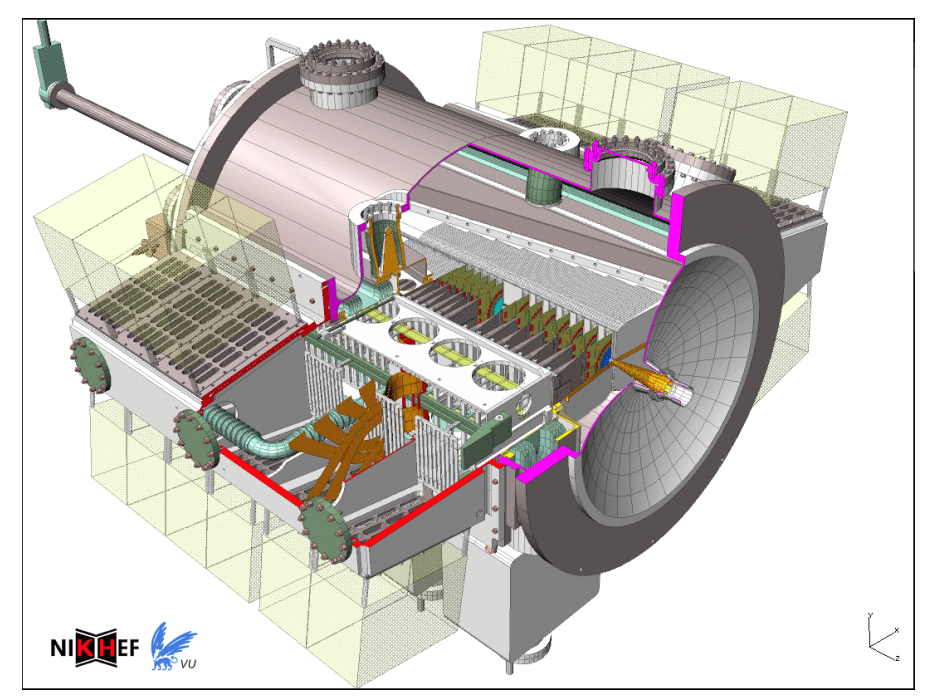

Figure 2: The LHCb VELO mechanical configuration.

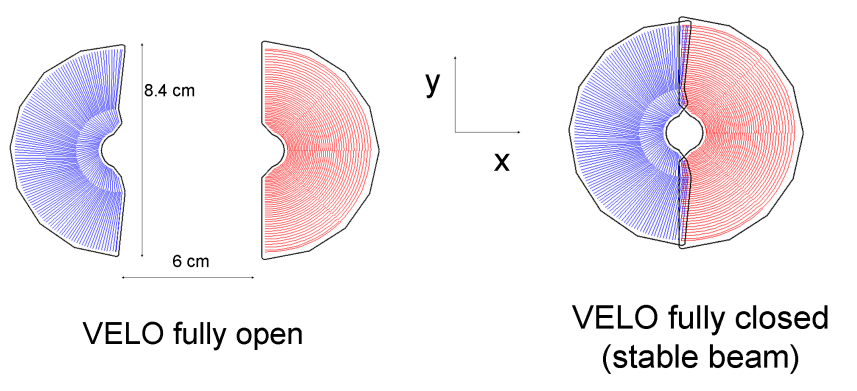

Figure 3: The LHCb VELO in parking position and closed around the beam. 


\section{The Tracker Turicensis (TT)}

The Tracker Turicensis (TT) is comprised of $p$-on- $n$ silicon microstrip sensors produced by HPK. The sensor thickness is $500 \mu \mathrm{m}$ with a strip pitch of $183 \mu \mathrm{m}$. The strip length goes up to 37 $\mathrm{cm}$, resulting in a capacitance of $60 \mathrm{pF}$. The TT has about $14 \mathrm{k}$ readout channels and an overall area of about $8 \mathrm{~m}^{2}$. During normal operations the sensor temperature is about $-8^{\circ} \mathrm{C}$ and the optimal hit resolution is $53.4 \mu \mathrm{m}$. The is placed in front of the $\mathrm{LHCb}$ dipole magnet and has four stereo layers as illustrated in Figure 4.

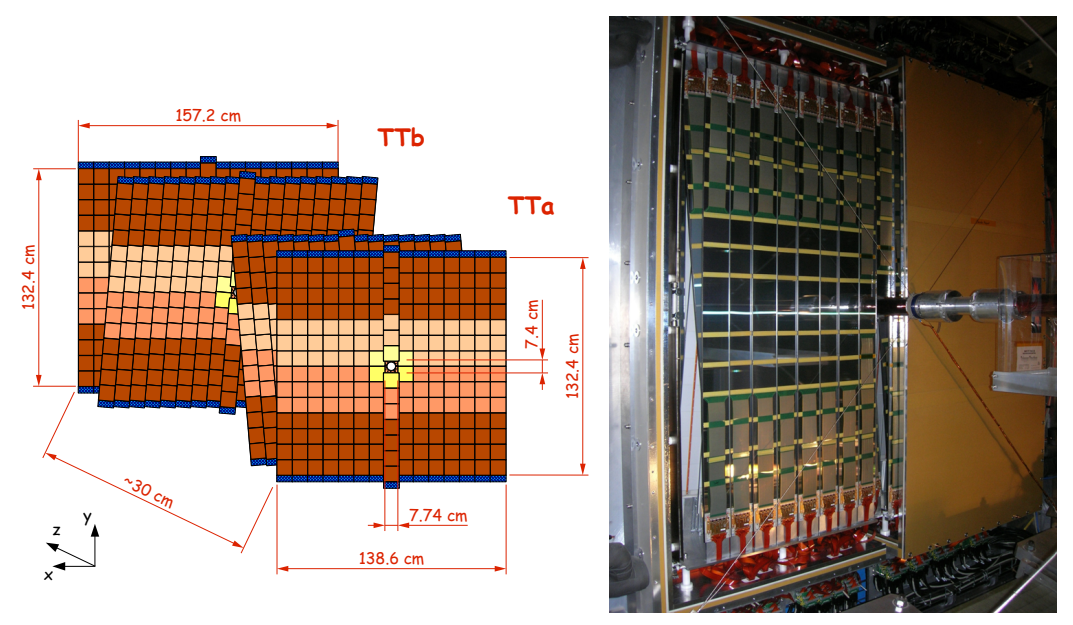

Figure 4: Schematic of TT stero layers (left) and picture of installed before closing TT (right).

\section{The Inner Tracker (IT)}

The LHCB Inner Tracker (IT) is comprised of 12 layers of HPK silicon microstrip sensors. The sensors have different widths and thicknesses. The $11 \mathrm{~cm}$ wide sensors have a thickness of $320 \mu \mathrm{m}$ and the $22 \mathrm{~cm}$ wide sensors have a thickness of $410 \mu \mathrm{m}$. The IT features a strip pitch of $198 \mu \mathrm{m}, 130 \mathrm{k}$ readout channels and $x u v x$ stereo angles of $0^{\circ},-5^{\circ},+5^{\circ}, 0^{\circ}$. The total silicon area of the LHCb IT is $4.2 \mathrm{~m}^{2}$ and the resolution is $54.9 \mu \mathrm{m}$. The layout and installation of the LHCb IT is illustrated in Figure 5.

\section{Radiation Damage}

Monitoring the effects of radiation damage is interesting on its own as it provides valuable input to the silicon detector community for the development of future detectors. For the LHCb silicon detectors, in particular for the VELO, it is essential to ensure the detector will be fully operational throughout the projected lifetime of the current LHCb experiment. Several methods are employed to monitor the radiation damage in the LHCb silicon detectors in order to crosscheck results and increase confidence in the operational parameters derived from these analyses. The methods to monitor radiation damage in the $\mathrm{LHCb}$ silicon detectors are: 

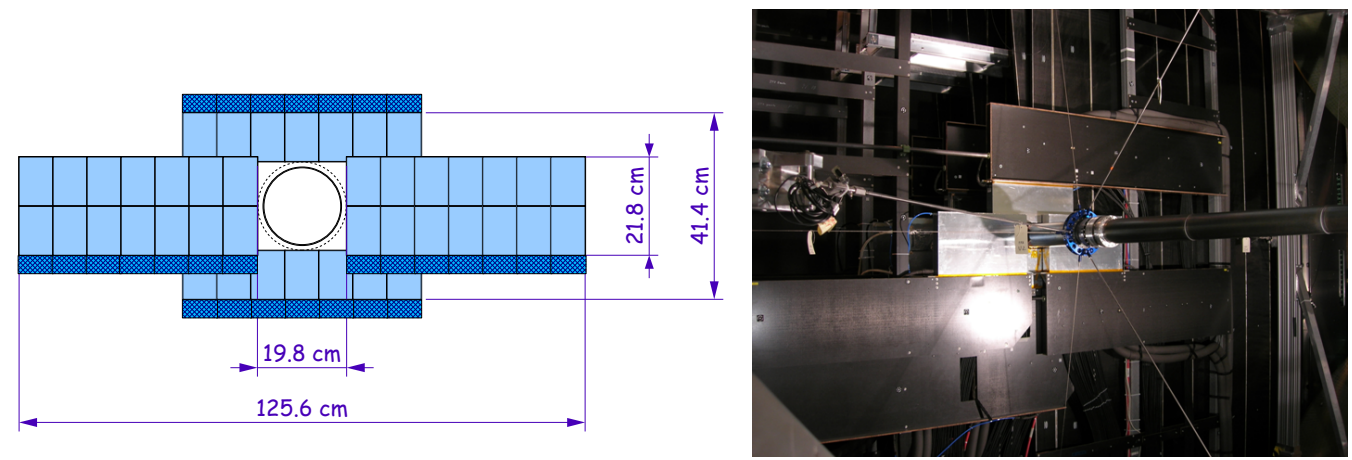

Figure 5: Schematic of IT layout (left) and picture of IT with beam pipe (right).

- Current vs. temperature (IT).

- Current vs. voltage (IV).

- Effective depletion voltage (EDV).

- Cluster finding efficiency (CFE).

The main difference in terms of operations between the different monitoring methods is whether they require colliding LHC beams or not. Special data-taking is required for the latter two monitoring methods in the form of a special procedure called CCE (Charge Collection Efficiency) scan. Collecting this special data cuts into physics data-taking and should therefore be minimised. Due to this constraint, acquiring the all the data to monitor radiation damage can not be fully automated and requires constant communication between the LHCb sub-detectors to be efficient. The CCE scans are therefore always taken with the VELO, TT and IT simultaneously. Others, like the IT scan, can only be performed during LHC technical stops and require experts for operating the cooling systems. The IV scans are very quick and can be performed easily in between LHC fills. For Run 2 the VELO has implemented an automatic procedure that records an IV scan at the end of each fill.

The IV data from the VELO and the TT is displayed in Figure 6 and Figure 7, respectively. After calibration of the temperature sensors the measured changes in bulk current due to accumulated radiation damage is in good agreement with the prediction. This allows to validate the predicted fluence for each sensor, which is particularly important for the VELO as it feeds into the projection of required bias voltages for Run 2. Figure 8 shows the predicted and measured leakage currents depending on their position. The predicted and measured values are in good agreement.

The CCE scan procedure, as illustrated in Figure 9, works like follows. For several patterns, shifting through the detector stations, a station is considered a device under test. For this station the bias voltage of the silicon sensors is varied while recording data from LHC collisions. The resulting data allows to determine the effective depletion voltage and cluster finding efficiency for all sensors in the LHCb silicon detectors by looking for clusters missing on tracks depending on the bias voltage. 


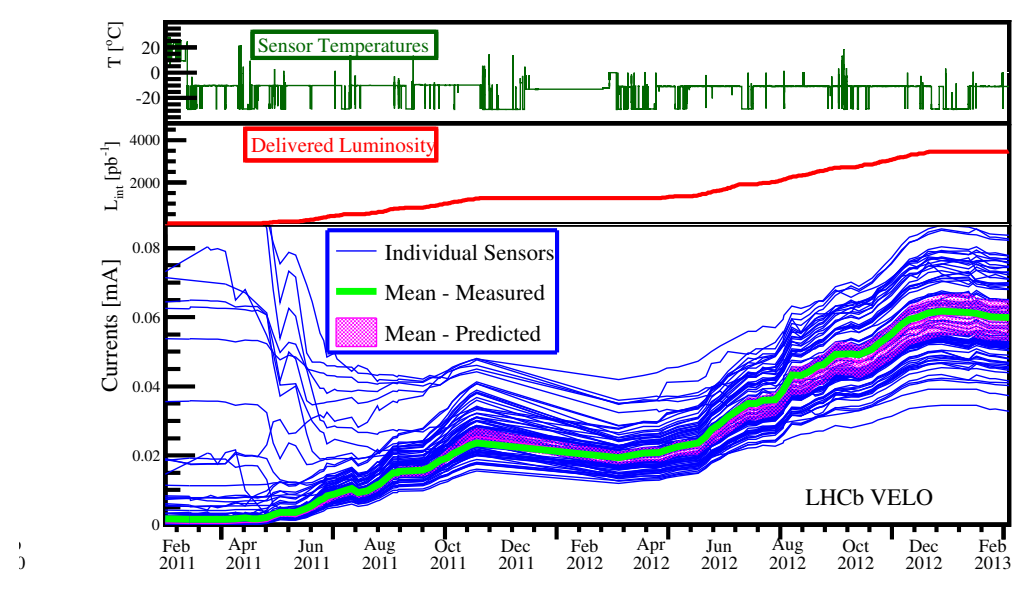

Figure 6: The leakage current recorded in the VELO sensors. The occasional drops are due to annealing (due to power cuts and such).

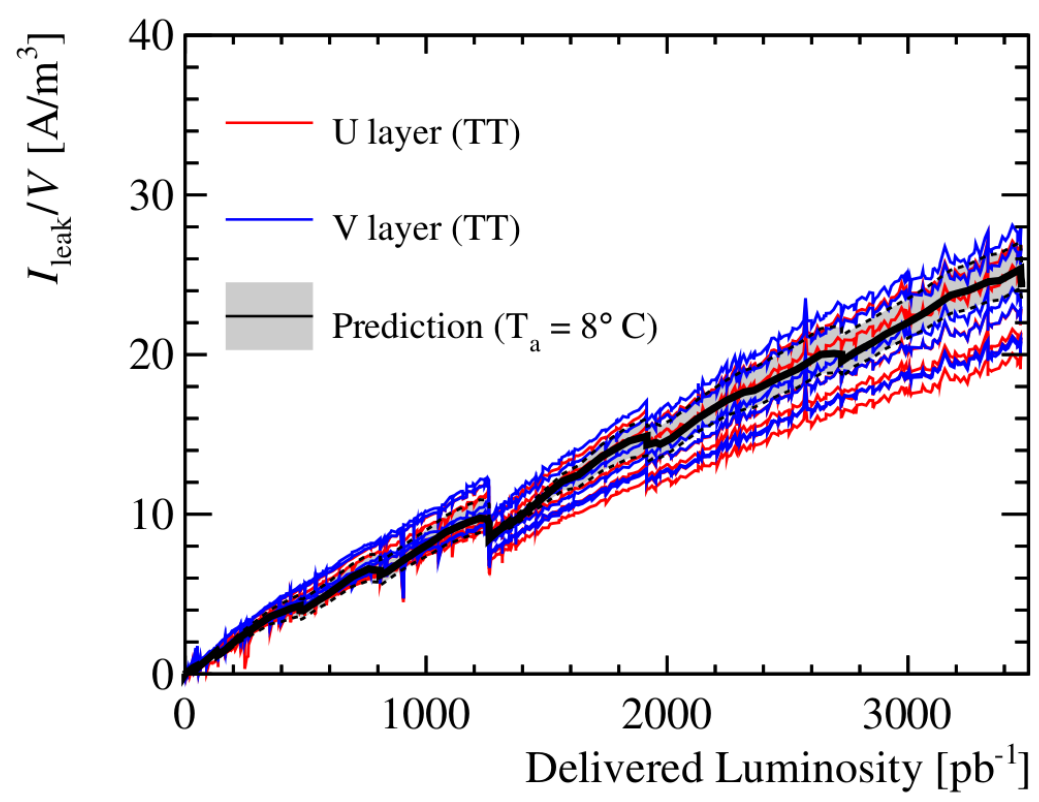

Figure 7: The leakage current recorded in the ST sensors. The data is normalised to $8^{\circ} \mathrm{C}$ and $E_{g}=1.21 \mathrm{eV}$.

The results are shown in Figure 10 for the VELO and Figure 11 for the ST. The measured effective depletion voltages are in good agreement with the Hamburg Model predictions. Using the results from the CCE scans, the bias voltages needed for efficient operation of the VELO during LHC Run 2 can be reliably predicted. For the most irradiated sensors closest to the interaction point the voltages have already been adapted accordingly. For the sensors the voltage has been increased from $150 \mathrm{~V}$ to $200 \mathrm{~V}$. The VELO also features one module with $n$-in- $p$ sensors which is now operated at $250 \mathrm{~V}$. Towards the end of Run 2 with an integrated luminosity of $10 \mathrm{fb}^{-1}$ the 


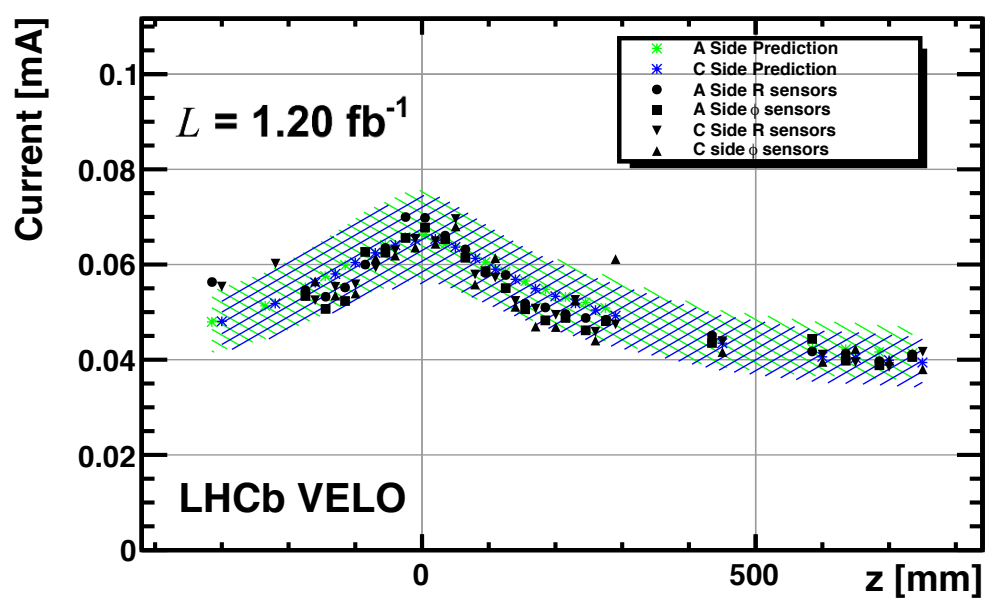

Figure 8: The leakage current in the VELO sensors depending on their distance from the interaction point. The measurements are in good agreement with the expectation from predicted fluences.

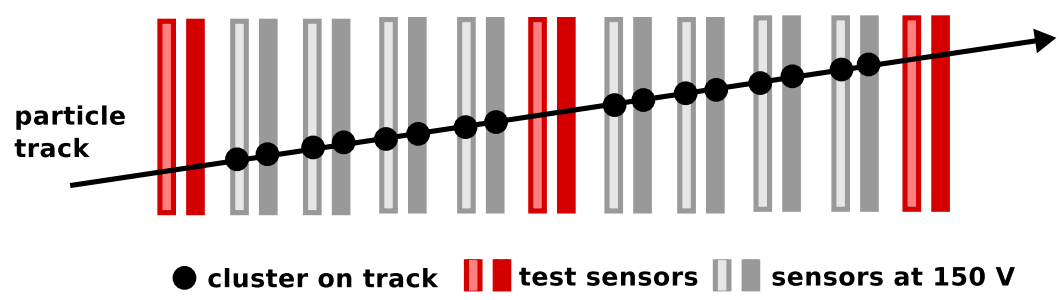

Figure 9: The VELO and ST CCE Scan method.

highest required voltage is expected to be $\sim 430 \mathrm{~V}$, well below the $500 \mathrm{~V}$ for which the system is qualified.

\section{Maintenance during LS1 and preparation for Run 2}

All major components of the $\mathrm{LHCb}$ silicon detector operational infrastructure have undergone scheduled maintenance and upgrades during LS1. The slow control software has been cleaned up and large parts were re-written to facilitate operations based on Run 1 experience. The VELO low voltage system has been completely refurbished to mitigate the risk of connector overheating on the low voltage bulk supplies. The VELO emergency spare has been moved back to the surface to comply with radiation protection procedures in the unlikely event it becomes necessary to install it. The VELO has been kept cool at a set-point of $-30{ }^{\circ} \mathrm{C}$ during the entire LS1, with the exception of brief periods of power outage and scheduled maintenance. An expert was on shift at all times to monitor the state of VELO cooling and vacuum system. The entire cooling plant for the ST has been replaced because its performance degraded over Run 1 due to lubricant contaminating the coolant. After the installation of a new beam pipe the IT could be moved to its nominal position. The IT has also been fitted with a position monitoring system which provides a more robust starting 


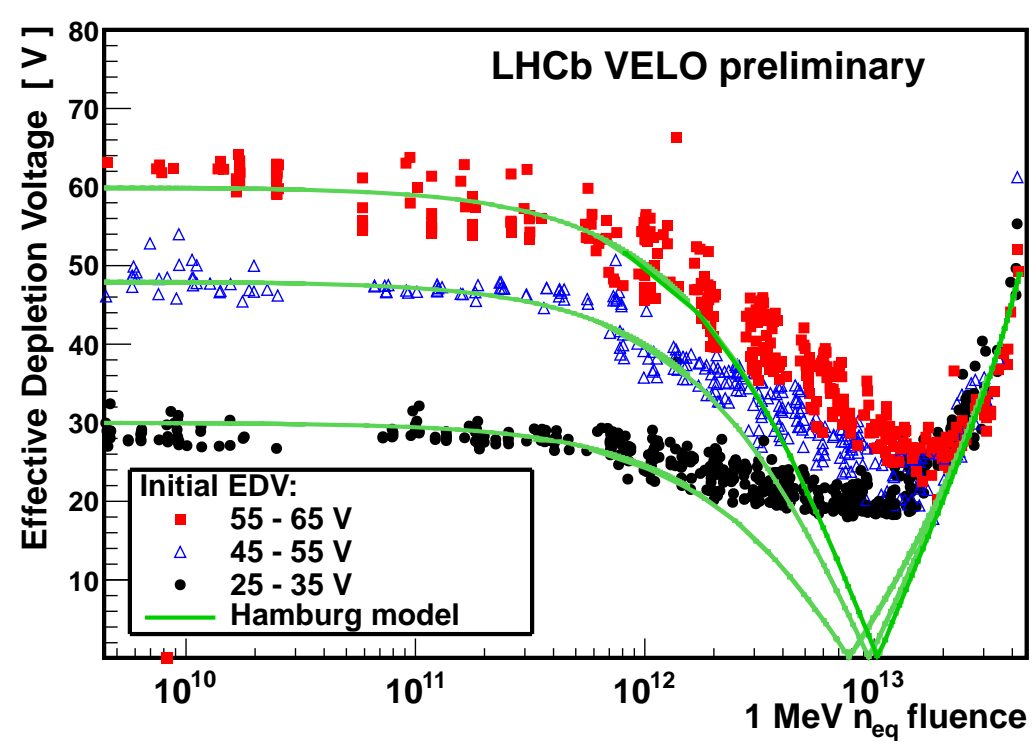

Figure 10: Effective depletion voltage versus fluence in the VELO sensors as determined from the CCE scan. The sensors closest to the interaction region have undergone type inversion. The comparison with the Hamburg Model shows good agreement.

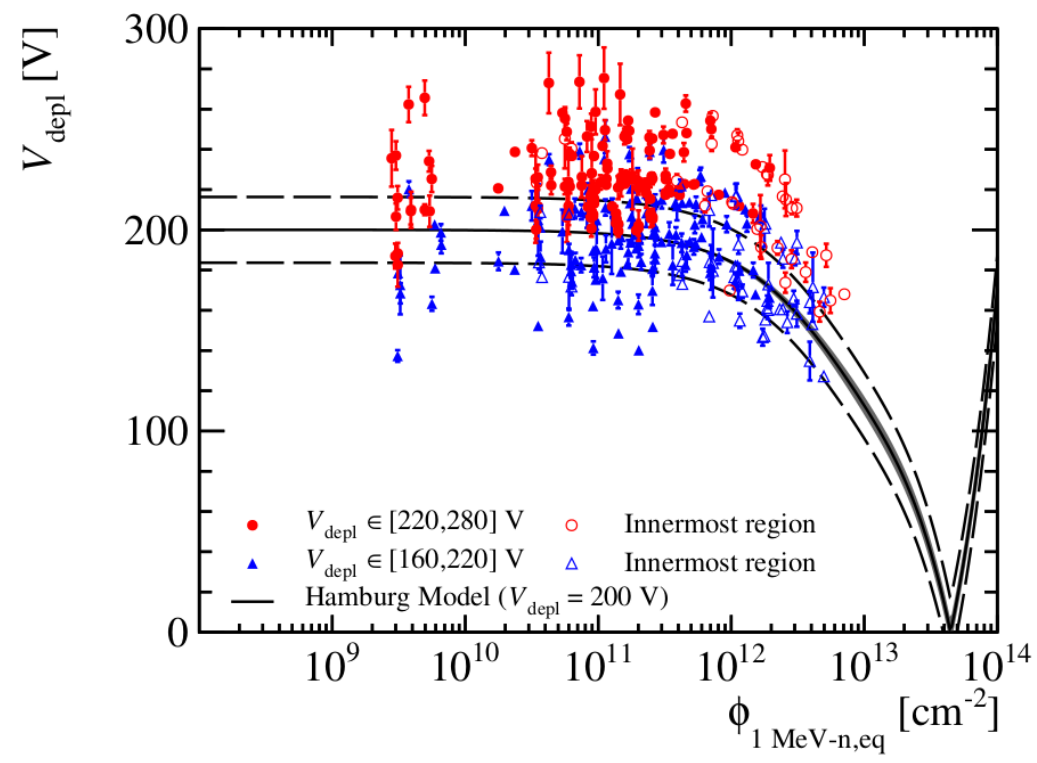

Figure 11: Effective depletion voltage versus fluence in the ST sensors as determined from the CCE scan. The data is in good agreement with the Hamburg Model. 
point for the software alignment procedure and allows to quickly spot movements due to changing magnet polarity.

In order to qualify new infrastructure components and calibration procedures, the silicon detectors have recorded data from shots on transfer line beam absorbers and beam gas interaction data. This was extremely useful and led to further improvements in some calibration procedures, in particular the time alignment of the silicon detectors. The LHCb silicon detectors were hence qualified and ready for data-taking well in advance of the start of Run 2. The VELO has implemented a new data quality (DQ) monitoring system with the aim to produce fully automated DQ reports after a period of training the criteria. Figure 12 shows the vertex summary page of the new tool for data recorded during an early fill of Run 2. Since the start of Run 2 the silicon detectors have recorded $223 p b^{-1}$ of integrated luminosity (see Figure 13).

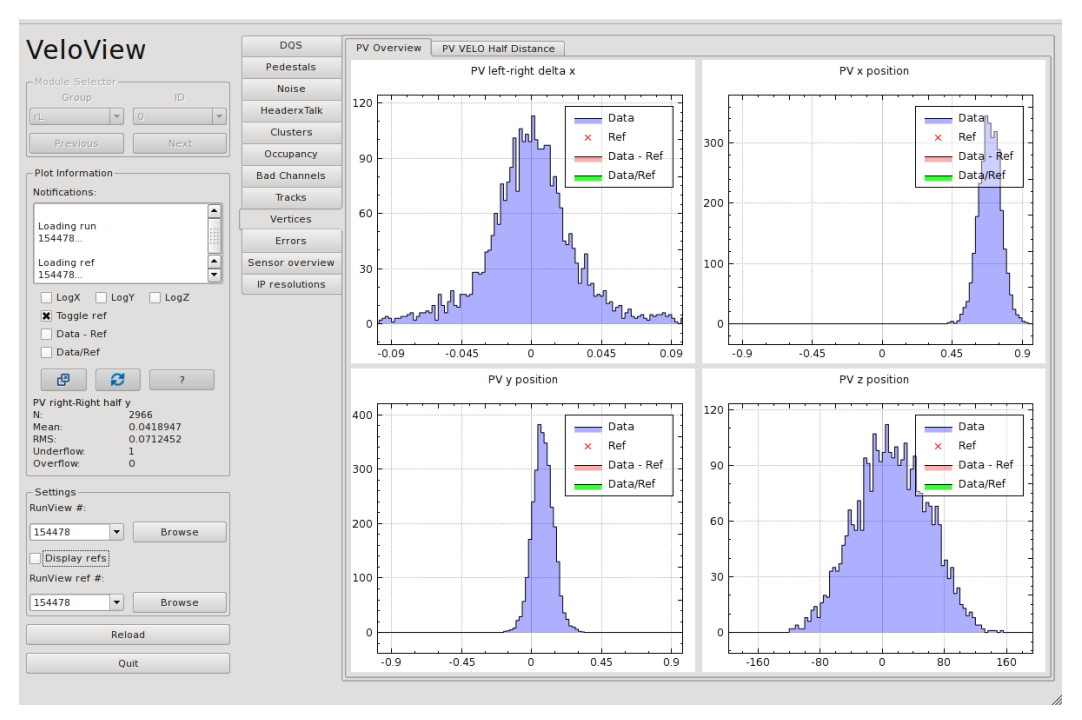

Figure 12: The vertex summary page of the new VELO DQ monitoring for an early fill of Run 2.

\section{Conclusion}

The LHCb silicon detectors have been kept safe and were well monitored during LS1. Many components of the operational infrastructure have undergone scheduled maintenance in preparation for LHC Run 2. The new infrastructure and calibration methods have been successfully tested by recording data from shots on the transfer line beam absorber and beam gas interaction in advance of physics data-taking. The radiation damage in the silicon detectors is well understood and monitored. Since the start of LHC Run 2 the silicon detectors have been efficiently operated and are well prepared to continue to do so throughout the entire LHC Run 2. 
LHCb Integrated Luminosity at p-p 6.5 TeV in 2015

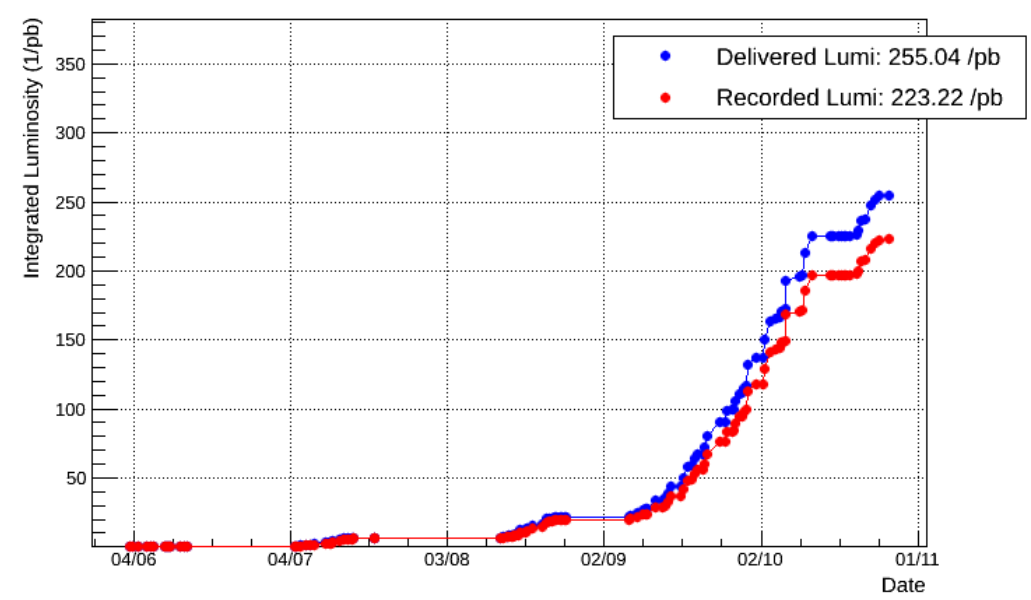

Figure 13: Delivered and recorded luminosity at LHCb for Run 2.

\section{References}

[1] The LHCb Collaboration, The LHCb Detector at the LHC, JINST 3 (2008) S08005

[2] The LHCb Collaboration, LHCb Detector Performance, Int. J. Mod. Phys. A 30 (2015) 1530022 , [arXiv:hep-ex/1412.6352]

[3] The LHCb VELO Group, Performance of the LHCb Vertex Locator, J. Instrum. 9 (2014) P09007, [arXiv:hep-ex/1405.7808]

[4] Hennessy, K., The LHCb Vertex Locator Replacement Detector - Electrical and Thermal Performance Studies, CERN LHCb-PUB-2013-004 (2013)

[5] Tobin, M. et al., The LHCb Silicon Tracker, Nucl. Instrum. Meth. A732 (2013) 168-172

[6] Elsasser, C. et al., The LHCb Silicon Tracker, JINST 9 (2014) C01009

[7] R. Arink et al., Performance of the LHCb Outer Tracker, JINST 9 (2014) P01002, [arXiv:hep-ex/1311.2893]

[8] The LHCb VELO Group, Radiation damage in the LHCb Vertex Locator, J. Instrum. 8 (2013) P08002, [arXiv:hep-ex/1302.5259] 